\title{
Light Asymmetric Dark Matter on the Lattice: SU(2) Technicolor with Two Fundamental Flavors
}

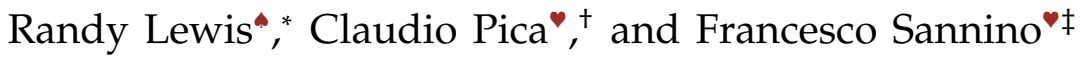 \\ -Department of Physics and Astronomy, \\ York University, Toronto, M3J 1P3, Canada and \\ - $\mathrm{CP}^{3}$-Origins $\mathcal{E}$ the Danish Institute for Advanced Study DIAS, \\ University of Southern Denmark, Campusvej 55, DK-5230 Odense M, Denmark
}

\begin{abstract}
The SU(2) gauge theory with two massless Dirac flavors constitutes the building block of several models of Technicolor. Furthermore it has also been used as a template for the construction of a natural light asymmetric, or mixed type, dark matter candidate. We use explicit lattice simulations to confirm the pattern of chiral symmetry breaking by determining the Goldstone spectrum and therefore show that the dark matter candidate can, de facto, be constituted by a complex Goldstone boson. We also determine the phenomenologically relevant spin-one and spin-zero isovector spectrum and demonstrate that it is well separated from the Goldstone spectrum.
\end{abstract}

Preprint: CP3-Origins-2011-28 \& DIAS-2011-15

\footnotetext{
* randy.lewis@yorku.ca

+ pica@cp3-origins.net

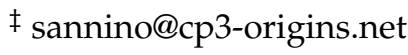




\section{INTRODUCTION}

The nature of dark matter (DM) is an important problem in modern physics. DM plays a key role in the formation of large structures and the evolution of the Universe. It is also widely expected to provide a link to physics beyond the Standard Model (SM). For these reasons much experimental, observational, and theoretical effort has been devoted to shedding light on DM. It is popular to identify DM with Weakly Interacting Massive Particles (WIMPs).

Many properties of a WIMP are not constrained by our current knowledge of DM, for example the WIMP may or may not be a stable particle [1] and it may or may not be identified with its antiparticle [2]. Asymmetric DM refers to a scenario where the WIMP's antiparticle has been annihilated away, leaving the WIMP itself as the observed DM. Asymmetric DM candidates were put forward in [2] as technibaryons, in [3] as Goldstone bosons, and subsequently in many diverse forms [4-10]. There is also the possibility of mixed DM [11], i.e. having both a thermally-produced symmetric component and an asymmetric one.

Null results from several experiments, such as CDMS [12] and Xenon10/100 [13, 14], have placed stringent constraints on WIMP-nucleon cross sections. Interestingly DAMA [15] and CoGeNT [16] have both produced evidence for an annual modulation signature for DM, as expected due to the relative motion of the Earth with respect to the DM halo. These results support a light WIMP with mass of order a few $\mathrm{GeV}$, which offers the attractive possibility of a common mechanism for baryogenesis and DM production. At first glance it seems that the WIMP-nucleon cross sections required by DAMA and CoGeNT have been excluded by CDMS and Xenon upon assuming spin-independent interactions between WIMPs and nuclei (with protons and neutrons coupling similarly to WIMPs), but a number of resolutions for this puzzle have been proposed in the literature [17-22]. Interestingly, new results from the CRESST-II experiment report signals of light DM [23].

A composite origin of DM is an intriguing possibility given that the bright side of the universe, constituted mostly by nucleons, is also composite. Thus a new stronglycoupled theory could be at the heart of DM. In this work we investigate, for the first time on the lattice, a technicolor-type extension of the SM expected to naturally yield a 
light DM candidate, as introduced in [8] and used in [22] to reconcile the experimental observations.

\section{A LIGHT DARK MATTER TEMPLATE: $S U(2)$ TECHNICOLOR WITH TWO DIRAC FLA- VORS}

Asymmetric DM requires at least one complex field with a (nearly) conserved Abelian quantum number. It would be exciting if the theory predicting the existence of this state is also directly involved with the breaking of electroweak symmetry. Therefore a natural candidate is a technicolor model. Most of the states of the theory are much heavier than a few $\mathrm{GeV}$, but if there are Goldstone bosons not absorbed by the longitudinal degrees of freedom of the SM massive gauge bosons, they become primary candidates for producing a natural hierarchy between the $\mathrm{GeV}$ and the $\mathrm{TeV}$ scale.

The minimal technicolor theory that breaks the electroweak symmetry and features a light DM state was constructed first in [24, 25]. This theory is $S U(2)$ technicolor with two Dirac flavors, which has global symmetry $S U(4)$ expected to break to $S p(4)$. Five Goldstone bosons are generated of which three are eaten by the SM gauge bosons and a complex one (a techni-diquark which is essentially a technibaryon of this $S U(2)$ theory) remains in the spectrum. This is our candidate for the light asymmetric DM particle.

The walking version of this model, known as Ultra Minimal Walking Technicolor (UMT), has been constructed in [8] and it contains, besides the fermions in the fundamental representation, also a Dirac fermion in the adjoint. For the present exploratory work, we begin with Section III of [8] but without the adjoint fermion. Moreover, when implementing our lattice simulations we make two additional modifications: all electroweak interactions are omitted from the lattice simulations because that physics is well-understood by perturbative methods, and explicit technifermion masses are added because lattice simulations with exactly massless fermions are not practical. The resulting Lagrangian is very simple

$$
\mathcal{L}=-\frac{1}{4} F_{\mu \nu}^{a} F^{a \mu \nu}+\bar{U}\left(i \gamma^{\mu} D_{\mu}-m\right) U+\bar{D}\left(i \gamma^{\mu} D_{\mu}-m\right) D,
$$

where $U$ and $D$ are the two techniquark fields having a common bare mass $m, F_{\mu \nu}^{a}$ is the field strength, and $D_{\mu}$ is the covariant derivative. The Dirac and technicolor indices of $U$ 
and $D$ are not shown explicitly.

Lattice simulations will be used to extrapolate to $m=0$, and in that limit the Lagrangian has a global $S U(4)$ symmetry corresponding to the four chiral fermion fields

$$
U_{L}=\frac{1}{2}\left(1-\gamma^{5}\right) U, \quad U_{R}=\frac{1}{2}\left(1+\gamma^{5}\right) U, \quad D_{L}=\frac{1}{2}\left(1-\gamma^{5}\right) D, \quad D_{R}=\frac{1}{2}\left(1+\gamma^{5}\right) D .
$$

For $m \neq 0$, the $S U(4)$ symmetry is explicitly broken to a remaining $S p(4)$ subgroup as follows. The Lagrangian from (1) can be rewritten as

$$
\mathcal{L}=-\frac{1}{4} F_{\mu v}^{a} F^{a \mu v}+i \bar{U} \gamma^{\mu} D_{\mu} U+i \bar{D} \gamma^{\mu} D_{\mu} D+\frac{m}{2} Q^{T}\left(-i \sigma^{2}\right) C E Q+\frac{m}{2}\left(Q^{T}\left(-i \sigma^{2}\right) C E Q\right)^{\dagger},
$$

where

$$
Q=\left(\begin{array}{c}
U_{L} \\
D_{L} \\
-i \sigma^{2} C \bar{U}_{R}^{T} \\
-i \sigma^{2} C \bar{D}_{R}^{T}
\end{array}\right), \quad E=\left(\begin{array}{cccc}
0 & 0 & 1 & 0 \\
0 & 0 & 0 & 1 \\
-1 & 0 & 0 & 0 \\
0 & -1 & 0 & 0
\end{array}\right),
$$

$C$ is the charge conjugation operator acting on Dirac indices, and the Pauli structure $-i \sigma^{2}$ is the standard antisymmetric tensor acting on color indices. Under an infinitesimal SU(4) transformation defined by

$$
Q \rightarrow\left(1+i \sum_{n=1}^{15} \alpha^{n} T^{n}\right) Q
$$

the Lagrangian (3) becomes

$$
\mathcal{L} \rightarrow \mathcal{L}+\frac{i m}{2} \sum_{n=1}^{15} \alpha^{n} Q^{T}\left(-i \sigma^{2}\right) C\left(E T^{n}+T^{n T} E\right) Q+\text { h.c. },
$$

where $T^{n}$ denotes the 15 generators of $S U(4)$ and $\alpha^{n}$ is a set of 15 constants. The only generators that leave the Lagrangian invariant are those that obey

$$
E T^{n}+T^{n T} E=0
$$

which is precisely the definition of an $S p(4)$ Lie algebra. From this, it is straightforward to derive the ten $S p(4)$ generators in a specific representation; see the appendix of [8].

For $m=0$ the Lagrangian retains the full SU(4) symmetry but, by analogy with the $S U(3)$ theory of QCD, one might expect dynamical symmetry breaking associated with the appearance of a nonzero vacuum expectation value,

$$
\langle\bar{U} U+\bar{D} D\rangle \neq 0 .
$$


Since this vacuum expectation value has the same structure as the terms containing $m$ in the Lagrangian, the dynamical breaking would also be $S U(4) \rightarrow S p(4)$. According to Noether's theorem, the five broken generators would be accompanied by five Goldstone bosons.

Of course this suggestion of dynamical symmetry breaking must be checked nonperturbatively using first-principles lattice simulations. As reported below, we have done so and our lattice simulations provide direct verification of this dynamical symmetry breaking.

There have been several previous lattice studies of $S U(2)$ gauge theory with fermions in the fundamental representation [26], mainly motivated by interest at nonzero chemical potential, but all of these studies relied on the staggered action where the number of fermions is a multiple of 4 . Our minimal technicolor theory requires $N_{f}=2$ and thus we use the Wilson action instead of staggered fermions. When studying chiral symmetry breaking scenarios with Wilson fermions attention must be paid to the presence, on a lattice, of the unphysical Aoki phase. For fixed gauge coupling, the Aoki phase is entered as the quark mass is reduced. An analytic discussion of the Aoki phase symmetries for this theory is provided in [27], and three lattice studies are also available [28-30]. For our present simulations, we avoid the Aoki phase and work exclusively in the physical phase. There have been very few previous lattice results reported for $S U(2)$ gauge theory with 2 fundamental fermions [30-32] and in each case the primary focus was on a different action (either $N_{f}>2$ or adjoint representation fermions). Our work is the first lattice study focused on the mass spectrum of the two-color two-flavor theory, which is the familiar technicolor template.

\section{LATTICE HADRON OPERATORS AND EFFECTIVE FIELD THEORY}

The creation operator for a meson is the Hermitian conjugate of its annihilation operator, and a set of local annihilation operators for mesons is

$$
\begin{aligned}
O_{\bar{U} D}^{(\Gamma)} & \equiv \bar{U}(x) \Gamma D(x), \\
O_{\bar{D} U}^{(\Gamma)} & \equiv \bar{D}(x) \Gamma U(x), \\
O_{\bar{U} U \pm \bar{D} D}^{(\Gamma)} & \equiv \frac{1}{\sqrt{2}}(\bar{U}(x) \Gamma U(x) \pm \bar{D}(x) \Gamma D(x)),
\end{aligned}
$$


where $\Gamma$ is a chosen Dirac structure. In this work we consider $\Gamma=1, \gamma^{5}, \gamma^{\mu}$, or $\gamma^{\mu} \gamma^{5}$. In lattice simulations, meson masses are extracted from the time dependence of correlation functions, for example

$$
\begin{aligned}
C_{\bar{u} D}^{(\Gamma)}\left(t_{x}-t_{y}\right) & =\sum_{\vec{x}} \sum_{\vec{y}} O_{\bar{u} D}^{(\Gamma)}(y)\left(O_{\bar{u} D}^{(\Gamma)}(x)\right)^{\dagger} \\
& =\sum_{\vec{x}} \sum_{\vec{y}} \operatorname{Tr}\left[\Gamma D(y) \bar{D}(x) \gamma^{0} \Gamma^{\dagger} \gamma^{0} U(x) \bar{U}(y)\right]
\end{aligned}
$$

where $\operatorname{Tr}[\cdots]$ denotes a trace over Dirac and color indices and we have dropped the vacuum expectation values for the propagators as explained in Appendix A.

Perhaps surprisingly, local diquark (i.e. baryon) correlation functions provide no new data in this theory. To understand why, notice that the available local diquark operators are

$$
\begin{aligned}
& O_{U D}^{(\Gamma)} \equiv U^{T}(x)\left(-i \sigma^{2}\right) C \Gamma D(x), \\
& O_{D U}^{(\Gamma)} \equiv D^{T}(x)\left(-i \sigma^{2}\right) C \Gamma U(x) .
\end{aligned}
$$

(Some operators containing $U^{T} \cdots U$ or $D^{T} \cdots D$ are identically zero.) The diquark correlation function is therefore

$$
\begin{aligned}
C_{U D}^{(\Gamma)}\left(t_{x}-t_{y}\right) & =\sum_{\vec{x}} \sum_{\vec{y}} O_{U D}^{(\Gamma)}(y)\left(O_{U D}^{(\Gamma)}(x)\right)^{\dagger} \\
& =\sum_{\vec{x}} \sum_{\vec{y}} \operatorname{Tr}\left[\Gamma D(y) \bar{D}(x) \gamma^{0} \Gamma^{\dagger} C^{\dagger}\left(-i \sigma^{2}\right)^{\dagger} \gamma^{0 T} \bar{U}^{T}(x) U^{T}(y)\left(-i \sigma^{2}\right) C\right]
\end{aligned}
$$

and this can be rewritten by using two properties of the charge conjugation operator: one for a Dirac matrix,

$$
\gamma^{\mu T}=-C \gamma^{\mu} C^{\dagger}
$$

and the other for the Wilson fermion matrix,

$$
C^{-1}\left(-i \sigma^{2}\right)^{-1}(U(y) \bar{U}(x))^{T} C\left(-i \sigma^{2}\right)=U(x) \bar{U}(y),
$$

to arrive at

$$
\begin{aligned}
C_{U D}^{(\Gamma)}\left(t_{x}-t_{y}\right) & =\sum_{\vec{x}} \sum_{\vec{y}} \operatorname{Tr}\left[\Gamma D(y) \bar{D}(x) \gamma^{0} \Gamma^{\dagger} \gamma^{0} U(x) \bar{U}(y)\right] \\
& =C_{\bar{U} D}^{(\Gamma)}\left(t_{x}-t_{y}\right)
\end{aligned}
$$


for any choice of $\Gamma$. This conclusion means that a lattice simulation will find numericallyidentical correlation functions, and thus identical masses, for the meson and diquark. We have verified this explicitly in the lattice simulations described below.

Notice that the degenerate pairs have equal angular momentum but opposite parities, for example

$$
\begin{gathered}
J\left(O_{U D}^{(\Gamma)}\right)=J\left(O_{\bar{U} D}^{(\Gamma)}\right) \\
P\left(O_{U D}^{(\Gamma)}\right)=-P\left(O_{\bar{U} D}^{(\Gamma)}\right) .
\end{gathered}
$$

This relationship for $J^{P}$ has also been mentioned, for example, in [33]. We must conclude that the three pseudoscalar meson Goldstone bosons are accompanied by two scalar diquark Goldstone bosons. This is in contrast to the identification made in [8], where it was assumed that all five Goldstones would be pseudoscalars. Despite this, the effective Lagrangian in [8] remains unaltered because all scalar and pseudoscalar particles were retained in that work with the correct assignment with respect to the broken and unbroken generators of the chiral symmetry group ${ }^{1}$.

Now that the five Goldstones have been identified, we can define an effective theory wherein those five are the only fields. Indeed, our lattice simulations (discussed below) indicate that the non-Goldstone scalar/pseudoscalar hadrons are even heavier than the vector mesons, making it quite natural to integrate all non-Goldstones out of the effective theory. The annihilation operators are conveniently collected into a compact form,

$$
\delta \mathcal{L}=\sum_{n}\left(Q^{T}\left(-i \sigma^{2}\right) C \Gamma T^{n} Q\right) \Phi^{(\Gamma) n}
$$

where $\Phi^{(\Gamma) n}$ is a generic name for the $n^{\prime}$ th particle with Dirac structure $\Gamma$. This $\delta \mathcal{L}$ represents the effective Lagrangian couplings for external fields $\Phi^{(\Gamma) n}$. For the special case of the five Goldstone bosons, we sum $n$ only over the five broken generators and we must choose $\Gamma=\gamma^{5}$. The result is

$$
\delta \mathcal{L}_{\mathcal{G}}=Q^{T}\left(-i \sigma^{2}\right) C \gamma^{5} \mathcal{G} Q
$$

where the matrix $\mathcal{G}$ contains the five Goldstone bosons (i.e. the pseudoscalar mesons $\Pi^{+}$,

\footnotetext{
${ }^{1}$ In practice the only change is the redefinition of $\widetilde{\Pi}_{U D}$ and $\widetilde{\Pi}_{\overline{U D}}$ at the underlying operator level with $\Pi_{U D}$ and $\Pi_{\overline{U D}}$ in [8].
} 
$\Pi^{-}, \Pi^{0}$ and the scalar diquarks $\left.\Pi_{U D}, \Pi_{\overline{U D}}\right)$ :

$$
\mathcal{G}=\frac{i}{2}\left(\begin{array}{cccc}
0 & \sqrt{2} \Pi_{U D} & \Pi^{0} & \sqrt{2} \Pi^{+} \\
-\sqrt{2} \Pi_{U D} & 0 & \sqrt{2} \Pi^{-} & -\Pi^{0} \\
-\Pi^{0} & -\sqrt{2} \Pi^{-} & 0 & -\sqrt{2} \Pi_{\overline{U D}} \\
-\sqrt{2} \Pi^{+} & \Pi^{0} & \sqrt{2} \Pi_{\overline{U D}} & 0
\end{array}\right) .
$$

The matrix $\mathcal{G}$ is closed under $S p(4)$ transformations: the Goldstone bosons form a fivedimensional representation of $S p(4)$. The matrix $\mathcal{G}$ is also identical to $M_{4}$ as defined by equation (22) of [8] after the non-Goldstone fields are removed from $\mathrm{M}_{4}$.

To summarize, an effective field theory for the five Goldstone bosons is obtained by using the matrix $\mathcal{G}$ in place of $M_{4}$ in [8]. The three pseudoscalar Goldstones $\left(\Pi^{ \pm}, \Pi^{0}\right)$ are responsible for electroweak symmetry breaking, and the two scalar Goldstones $\left(\Pi_{U D}\right.$, $\left.\Pi_{\overline{U D}}\right)$ are the DM candidate and its antiparticle.

\section{METHODS FOR NUMERICAL SIMULATIONS}

The standard Wilson action,

$$
\begin{aligned}
S_{W}= & \frac{\beta}{2} \sum_{x, \mu, v}\left(1-\frac{1}{2} \operatorname{ReTr}_{\mu}(x) U_{v}(x+\hat{\mu}) U_{\mu}^{+}(x+\hat{v}) U_{v}^{+}(x)\right)+\left(4+m_{0}\right) \sum_{x} \bar{\psi}(x) \psi(x) \\
& -\frac{1}{2} \sum_{x, \mu}\left(\bar{\psi}(x)\left(1-\gamma_{\mu}\right) U_{\mu}(x) \psi(x+\hat{\mu})+\bar{\psi}(x+\hat{\mu})\left(1+\gamma_{\mu}\right) U_{\mu}^{+}(x) \psi(x)\right),
\end{aligned}
$$

is used for this study of $S U(2)$ gauge theory with two mass-degenerate fermions in the fundamental representation. Configurations were generated using the HMC algorithm as implemented in the HiRep code [34]. A total of 12 ensembles were created, corresponding to six different bare quark masses $m_{0}$ for each of two different gauge couplings $\beta$; see Table I] for details. We note that [30] also contains some simulations of this theory at $\beta=2.0$ and our findings for the pseudoscalar meson mass and PCAC quark mass are consistent with that paper. For other parameter choices, see [31] and [32]. In the present study, all lattices are $L^{3} \times T=16^{3} \times 32$ with periodic boundary conditions in each direction. Every ensemble contains 35 configurations separated by 20 unused configurations after an initial thermalization of 320 configurations. Figures 1 and 2 display the fluctuations in the average plaquette within selected Markov chains. 
TABLE I: Numerical values of the gauge coupling and the bare quark mass used to generate the 12 ensembles of this project.

\begin{tabular}{ll}
\hline$\beta$ & $m_{0}$ \\
\hline 2.0 & $-0.85,-0.90,-0.94,-0.945,-0.947,-0.949$ \\
2.2 & $-0.60,-0.65,-0.68,-0.70,-0.72,-0.75$ \\
\hline
\end{tabular}

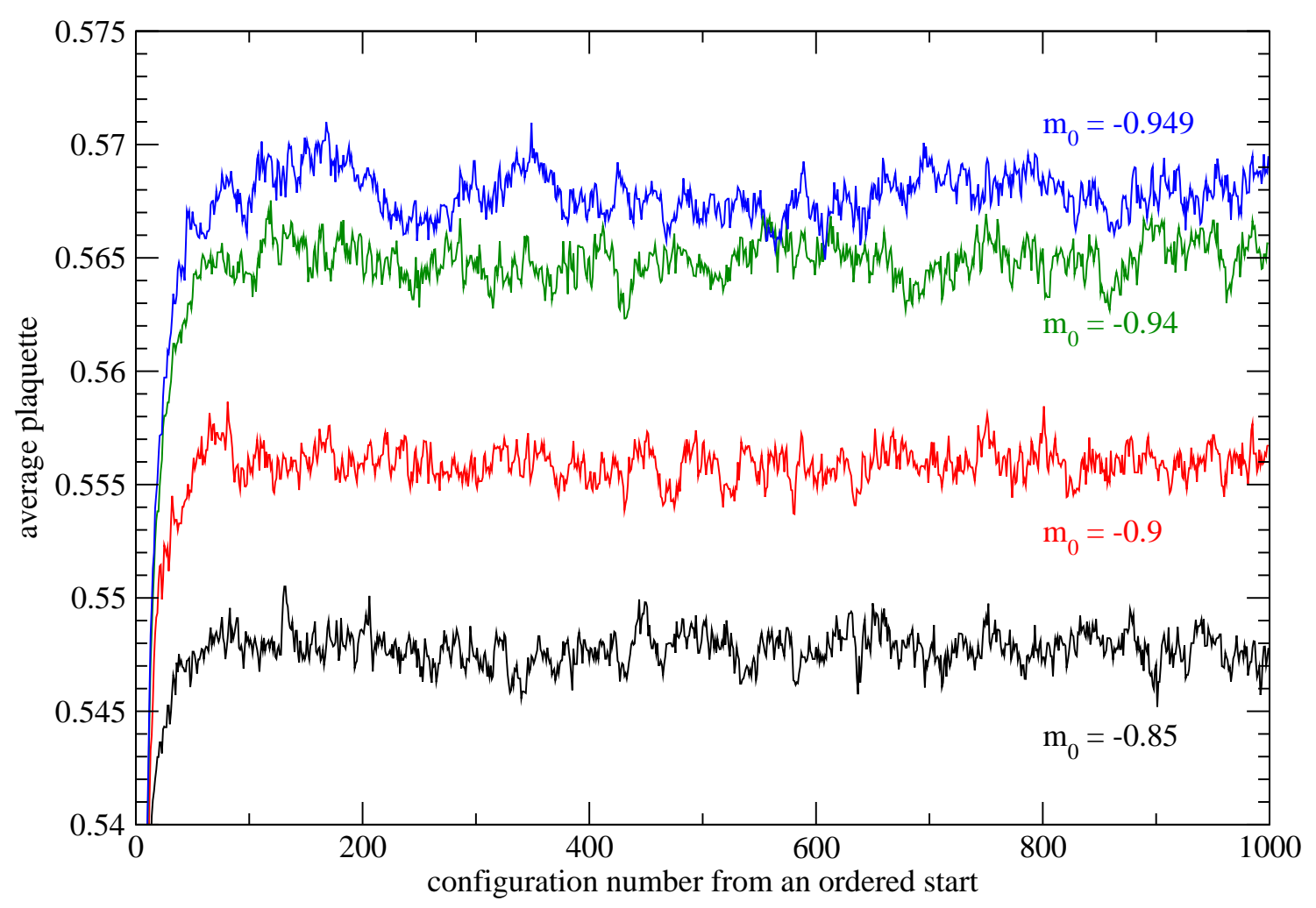

FIG. 1: Evolution of the average plaquette for selected bare masses at $\beta=2.0$. Our ensemble of 35 configurations is comprised of those numbered 320, 340, 360, . 1000.

Quark propagators are created from wall sources built of random $U(1)$ phases at every lattice site on one chosen time step, $t_{i}$. Inversions are performed with the standard BiCGstab algorithm. To reduce statistical fluctuations, correlation functions are averaged over all lattice times $0 \leq t_{i} \leq 31$. Correlation functions then depend on the time $t_{f}$ corresponding to the annihilation operator. This annihilation operator is local and summed 


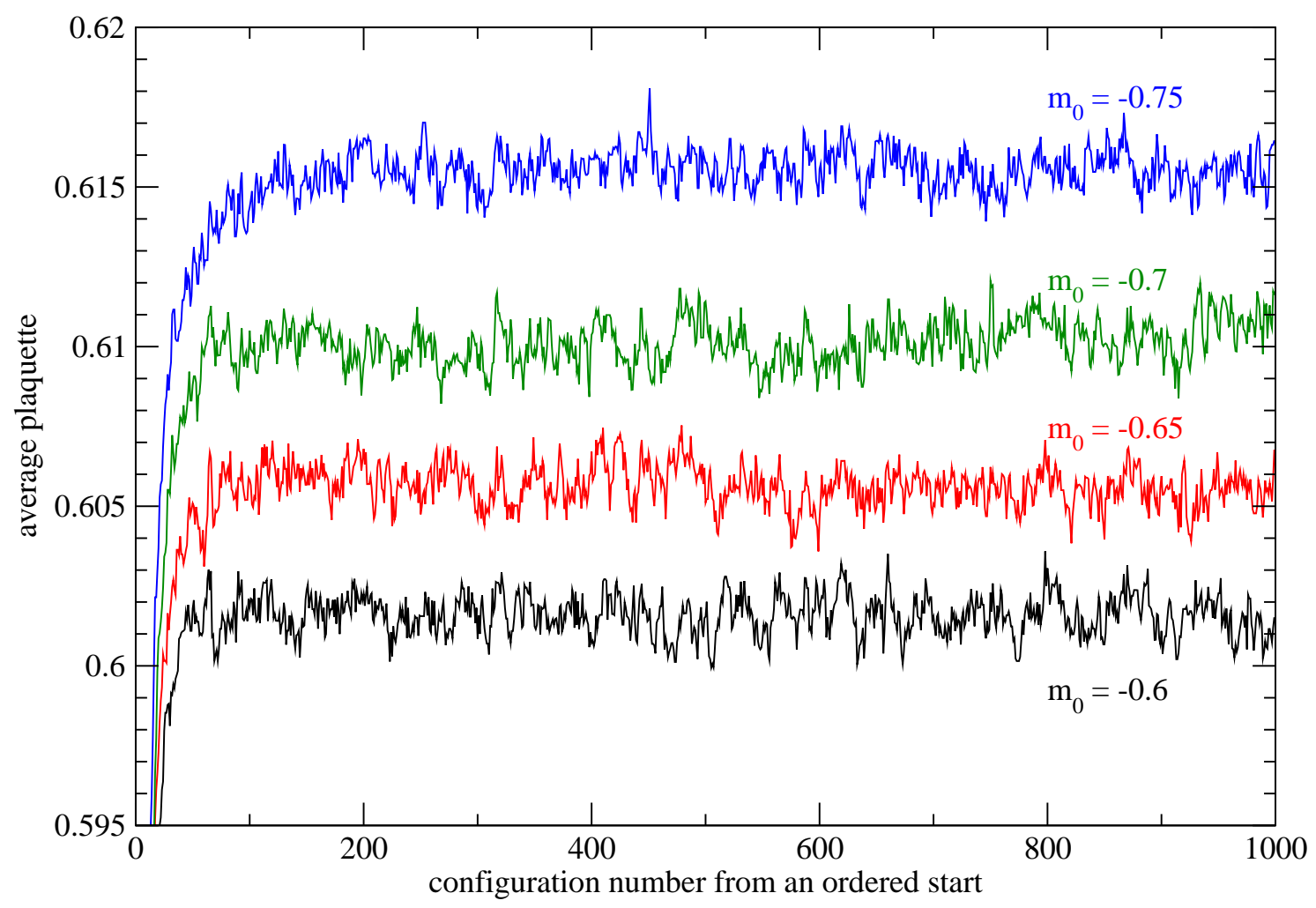

FIG. 2: Evolution of the average plaquette for selected bare masses at $\beta=2.2$. Our ensemble of 35 configurations is comprised of those numbered 320, 340, 360, . 1000.

over all spatial sites to produce a zero-momentum hadron.

Our multi-state fits,

$$
C(t)=\sum_{j=1}^{n} a_{j} \cosh \left(m_{j}\left(t-\frac{T}{2}\right)\right)
$$

use every nonzero time separation $t=t_{f}-t_{i}>0$, which avoids the subjectivity of choosing a fitting window. Pseudoscalar, vector and temporal-axial $\left(A_{4}\right)$ correlators are fitted to three states, $n=3$ in (22); scalar and spatial-axial $\left(A_{1}, A_{2}, A_{3}\right)$ correlators use $n=2$. Statistical uncertainties are produced from each 35 -configuration ensemble by creating 150 bootstrap ensembles (having 35 configurations each).

Isovector hadrons are sufficient for most topics addressed in this work, but isoscalars are used to study a specific issue. Isoscalar operators require the computation of singlesite propagators, i.e. quark propagators that begin and end on a single lattice site. In 


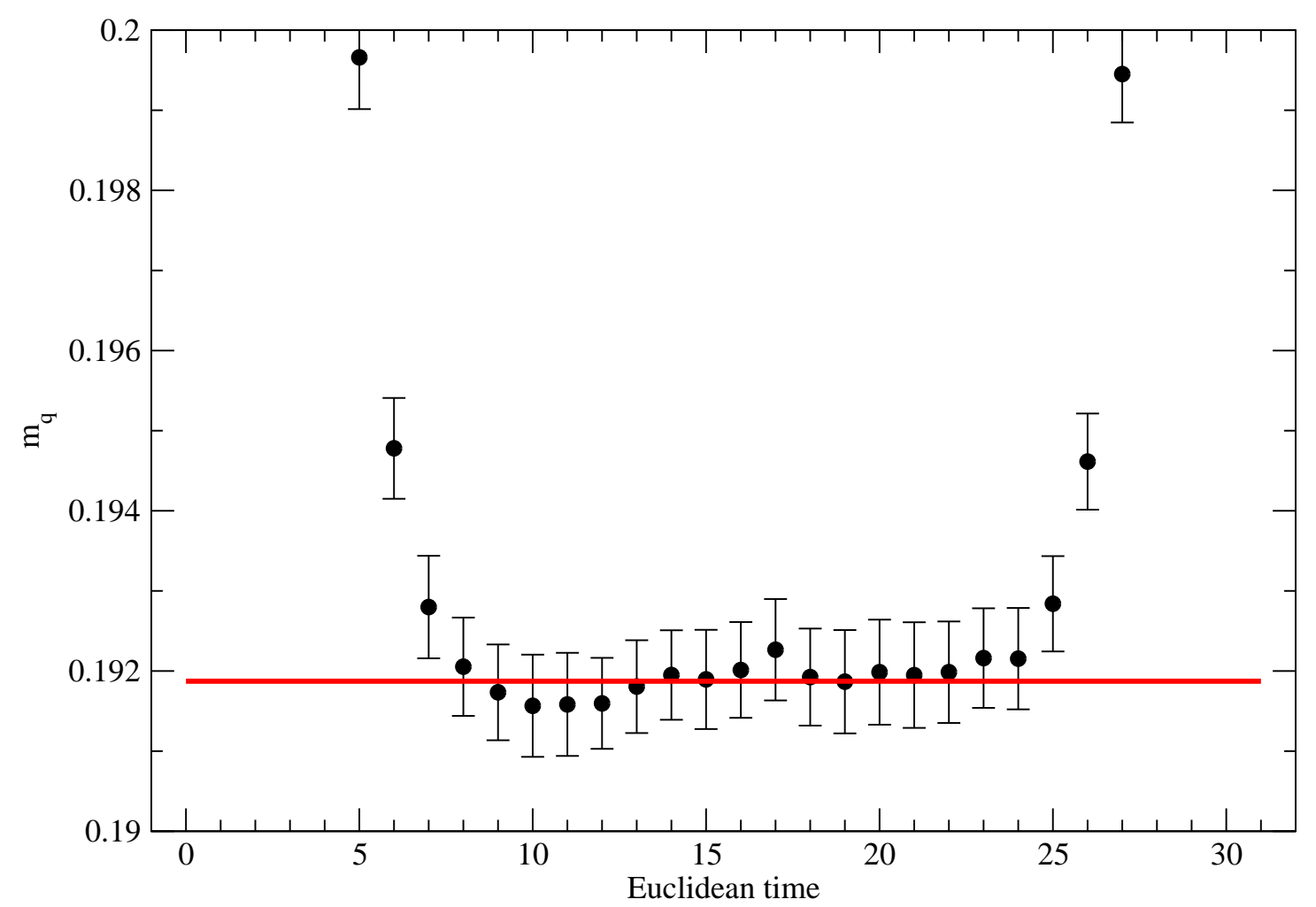

FIG. 3: The $t$ dependence from the right-hand side of 23 for the case of $\beta=2.2$ and $m_{0}=-0.75$. The horizontal line is a fit to Euclidean times between 10 and 22 inclusive.

practice, to obtain a signal requires an average over many sites, so isoscalar studies are expensive. Improved methods have recently been developed [35, 36], but the needs of our present project are fulfilled by a simpler tactic. We choose our "most physical" ensemble ( $\beta=2.2$ and $\left.m_{0}=-0.75\right)$ and calculate 2048 single-site propagators, that is 64 single-site propagators spread uniformly across each lattice time step.

Two additional quantities that can be derived from two-point correlation functions are also valuable for the present work. One is the PCAC quark mass defined by

$$
m_{q}=\lim _{t \rightarrow \infty}\left(\frac{\left\langle A_{4}(t+1) P(0)\right\rangle-\left\langle A_{4}(t-1) P(0)\right\rangle}{4\langle P(t) P(0)\rangle}\right),
$$

and in practice we can average over time separations satisfying $12 \leq t \leq 20$. Figure 3 shows the example of $\beta=2.2$ and $m_{0}=-0.75$. The other additional quantity is the Goldstone 
boson decay constant that we extract from a simultaneous fit to three correlators [37],

$$
\begin{aligned}
\left\langle A_{4}(t) A_{4}(0)\right\rangle & =\sum_{j=1}^{n} \frac{2 m_{j}}{\sqrt{L^{3}}}\left(\frac{f_{j}}{Z_{A}}\right)^{2} \cosh \left(m_{j}\left(t-\frac{T}{2}\right)\right), \\
\left\langle A_{4}(t) P(0)\right\rangle & =\sum_{j=1}^{n}\left(\frac{m_{j}}{2 m_{q} Z_{P}}\right) \frac{2 m_{j}}{\sqrt{L^{3}}}\left(\frac{f_{j}}{Z_{A}}\right)^{2} \cosh \left(m_{j}\left(t-\frac{T}{2}\right)\right), \\
\langle P(t) P(0)\rangle & =\sum_{j=1}^{n}\left(\frac{m_{j}}{2 m_{q} Z_{P}}\right)^{2} \frac{2 m_{j}}{\sqrt{L^{3}}}\left(\frac{f_{j}}{Z_{A}}\right)^{2} \cosh \left(m_{j}\left(t-\frac{T}{2}\right)\right),
\end{aligned}
$$

where $L=16$ and $T=32$. As stated above, we use $n=3$ and fit all time separations except $t=0$. The fitting parameters are $m_{j}, m_{j} /\left(m_{q} Z_{P}\right)$, and $f_{j} / Z_{A}$. Notice that such fits only provide the decay constant divided by its renormalization constant $Z_{A}$. Since $Z_{A}$ will approach unity in the continuum limit, we will study the ratio $f_{j} / Z_{A}$ rather than $f_{j}$ itself in this exploratory study.

\section{RESULTS FROM NUMERICAL SIMULATIONS}

As familiar from QCD, the axial Ward-Takahashi identity incorporates a partiallyconserved axial vector current (PCAC) which defines a renormalized quark mass in terms of the axial current's derivative. Use of the explicit definition in (23) produces the relationship between renormalized and bare quark masses as plotted in Fig. 4. Over our range of bare masses, the PCAC mass is seen to be effectively linear in the bare mass.

Goldstone bosons should be massless when the renormalized quark mass is zero, and should approach that limit according to

$$
m_{\Pi} \propto \sqrt{m_{q}} .
$$

The lattice data confirm this behavior, as shown in Fig. 5. At $\beta=2.0$ our entire range of masses satisfies (27). At $\beta=2.2$ our range of mass values is broad enough that the two heaviest quarks show curvature coming from corrections to (27), and our lightest quark displays a finite-volume correction. We wish to emphasize that these plots in Fig. 5 are obtained using any of the five operators from (9) and (11). All five Goldstone bosons are exactly degenerate. No single-site propagators were needed for computing these correlations functions. 

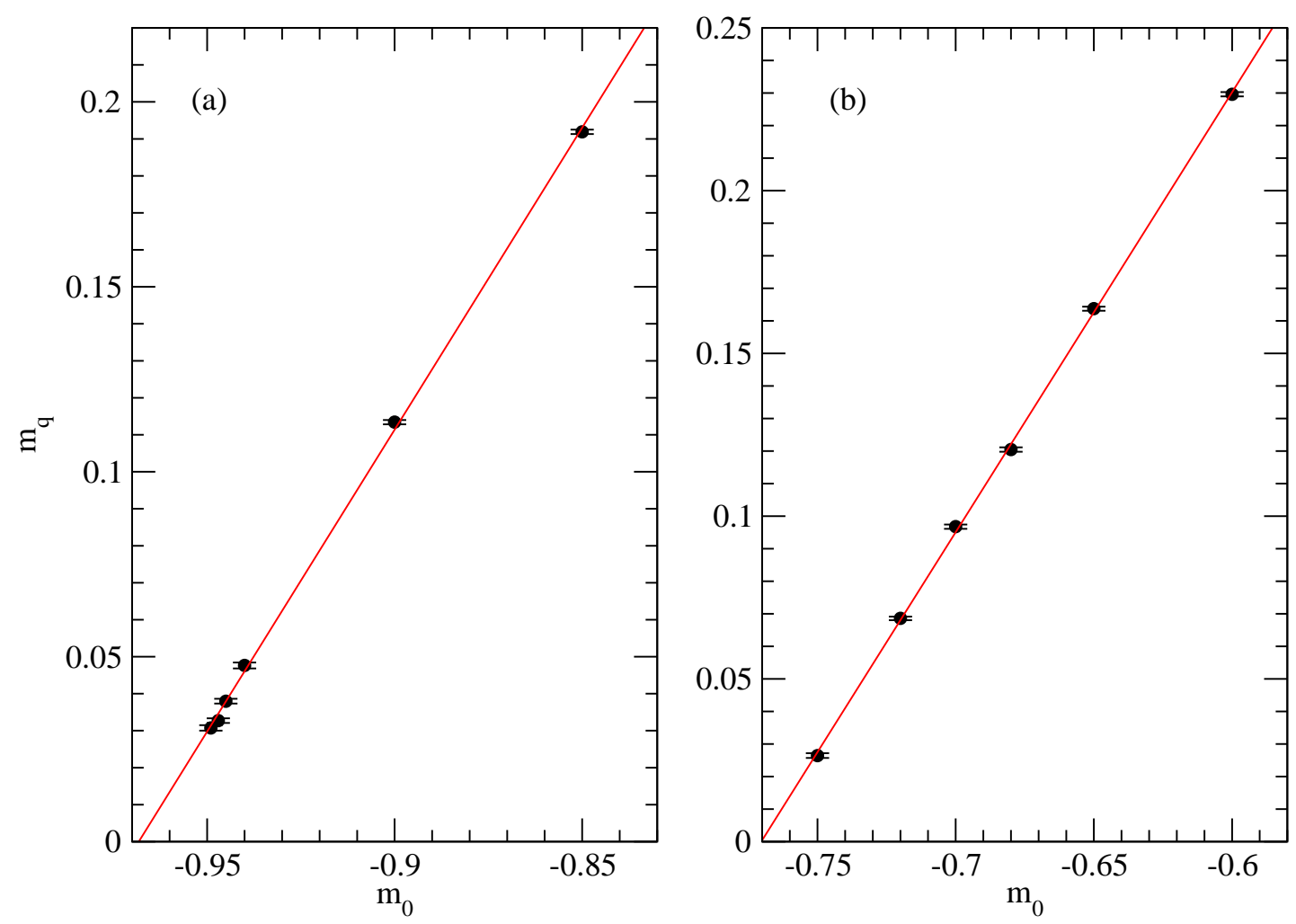

FIG. 4: The PCAC quark mass as a function of the bare quark mass for (a) $\beta=2.0$ and (b) $\beta=2.2$. In each case, the line is a fit to all six data points.

The physical scale of technicolor can be specified by requiring that the Goldstone decay constant matches the observed electroweak energy scale,

$$
f_{\Pi} \approx 246 \mathrm{GeV}
$$

Comparison to a lattice determination of $f_{\Pi}$ would thus provide a direct interpretation of the physical scale for each lattice simulation. Up to the renormalization constant $Z_{A}$, we can produce these lattice results by using (24,26), as displayed in Fig. 6. A curvature is clearly visible in these plots of $f_{\Pi} / Z_{A}$ versus $m_{q}$, and for our purposes it is sufficient to notice that $f_{\Pi} / Z_{A} \sim O(0.1)$ in our simulations, i.e. an order of magnitude below the lattice cut-off.

Figure 7, besides displaying again the Goldstone isovector spectrum, can be used to verify that non-Goldstone type states, such as the scalar and spin one isovector particles, 

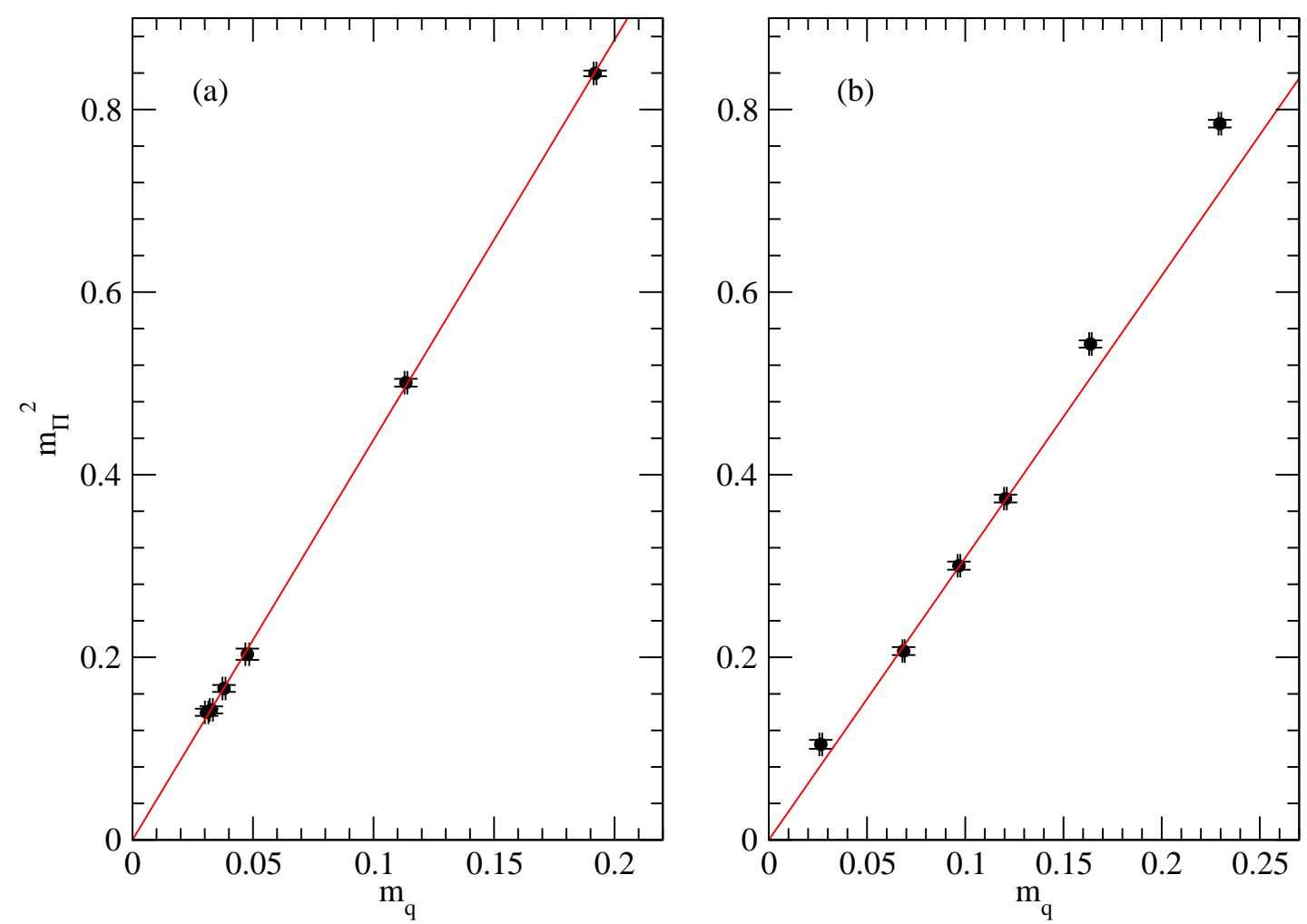

FIG. 5: The Goldstone boson mass squared as a function of the PCAC quark mass for (a) $\beta=2.0$ and (b) $\beta=2$.2. In (a) the line is a one-parameter fit to all six data points, but in (b) the line is a fit to the three points within $0.05<m_{q}<0.15$.

remain massive in the $m_{q}=0$ limit. Much like QCD, the vector meson mass is nearly an order of magnitude larger than $f_{\Pi}$, but significantly lighter than the isovector scalar and axial vector mesons. Lattice artifacts are presumably sizable for masses $m \gtrsim 1$, but our results do extend into the region where all masses are less than 1 . Notice the exact degeneracy between mesons and diquarks of opposite parity, as anticipated in Section III.

Although isoscalar correlation functions bring the major challenge of single-site propagators, we want to consider the specific case of the isoscalar pseudoscalar meson. Our goal is to verify explicitly that this meson is not an additional Goldstone particle. Figure 8 displays the results. Panel (a) is the correlation function for the isovector, i.e. the quarkconnected diagram only. A multi-exponential fit to those data produce the corresponding 

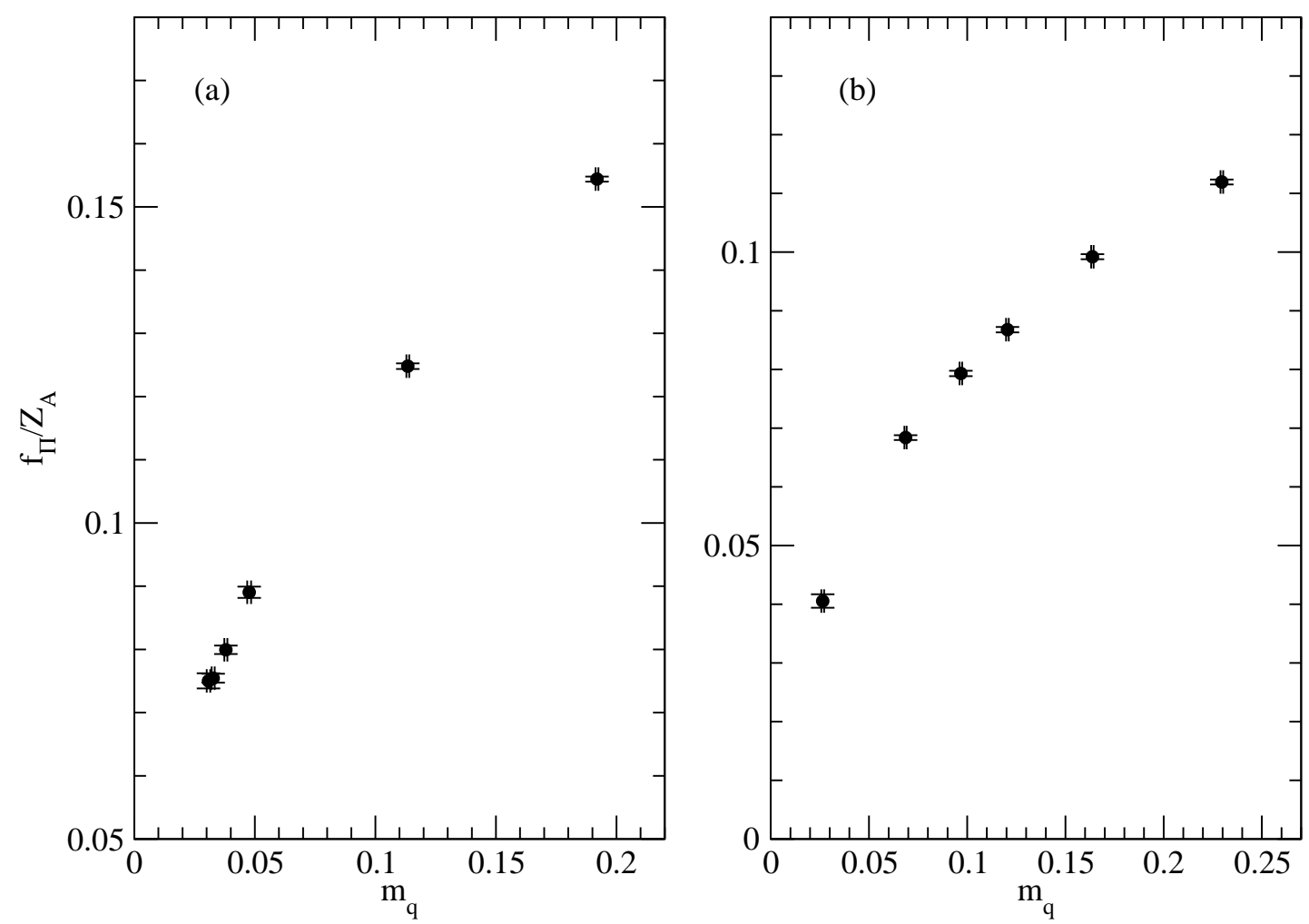

FIG. 6: The Goldstone boson decay constant as a function of the PCAC quark mass for (a) $\beta=2.0$ and (b) $\beta=2.2$.

data point in Fig. 5. Panel (b) is the contribution from the quark-disconnected diagram built from a pair of single-site quark propagators. Panel (c) is obtained from the upper two panels according to "(c)=(a)-2(b)", where the factor of 2 accounts for $\bar{U} U$ plus $\bar{D} D$ contributions and the minus sign accounts for anticommuting fermions. In panel (c), data at all timesteps between 7 and 24 are statistically consistent with zero, and time steps near the source at $t=0$ correspond to states much heavier than the Goldstones. Thus we see how the quark-disconnected diagrams have canceled the Goldstone signal out of the isoscalar. This is the expected conclusion since the symmetry breaking $S U(4) \rightarrow S p(4)$ requires exactly five Goldstone bosons. 


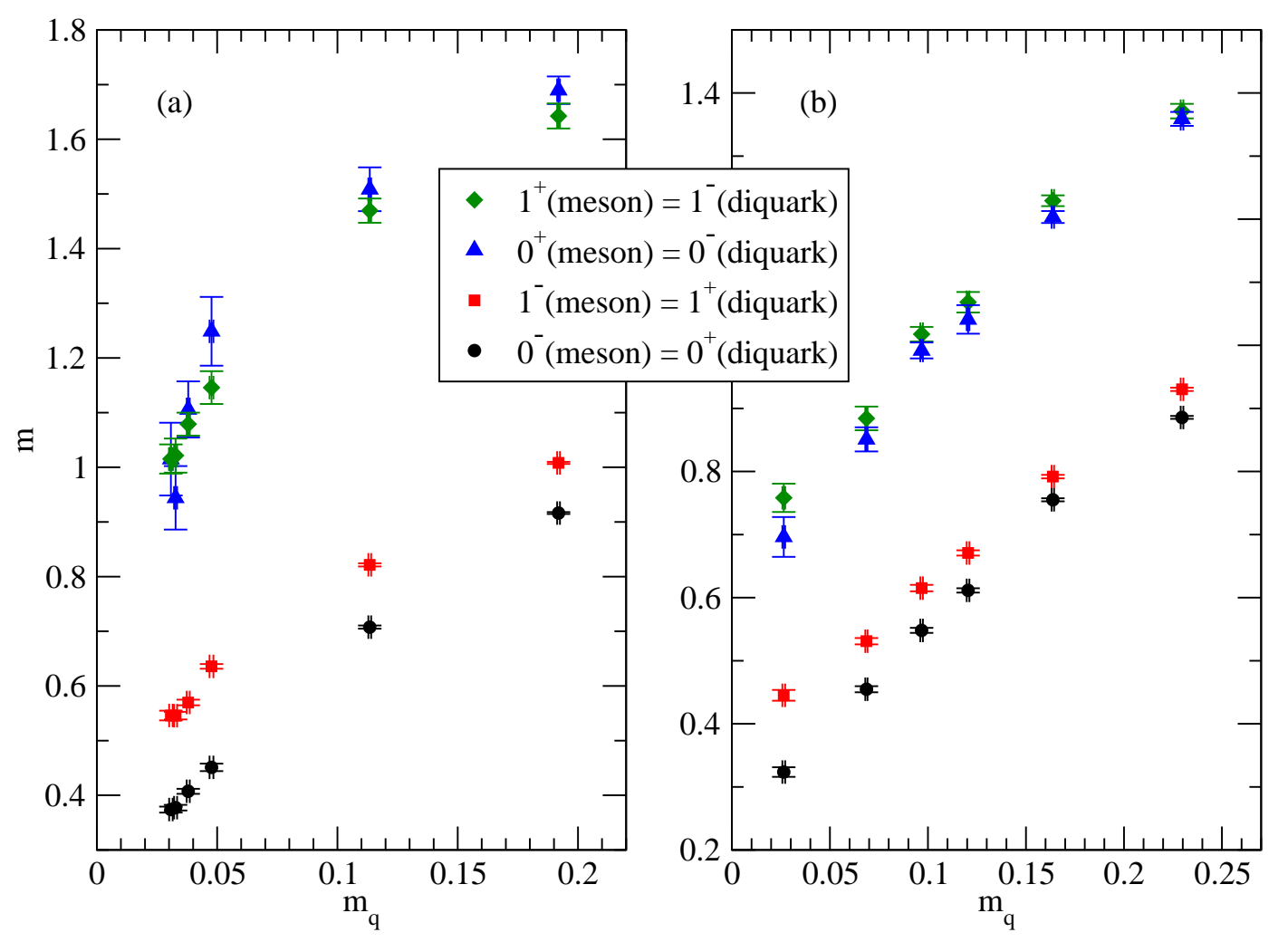

FIG. 7: Mass spectrum of isovector particles, with various $J^{P}$ quantum numbers, as a function of the PCAC quark mass for (a) $\beta=2.0$ and (b) $\beta=2.2$.

\section{CONCLUSIONS}

From many previous studies [2-11] the lightest neutral technibaryon has become a classic prototype for asymmetric DM, and [3, 4, 8, 22] focused on the possibility of that technibaryon being a pseudo-Goldstone boson. In this work, we have performed lattice simulations of $S U(2)$ gauge theory with two techniquarks which has been used as a template for the construction of composite light DM models [8, 22]. For the first time, we verify the existence of the pseudo-Goldstone state to identify with the composite DM candidate. In fact, besides the standard three Goldstone bosons, to be eaten up by the SM gauge bosons, we observe two more Goldstone technibaryons. These latter states are scalars rather than pseudoscalars. The masses of other phenomenologically relevant technihadrons were found to be an order of magnitude heavier than the Goldstone decay 

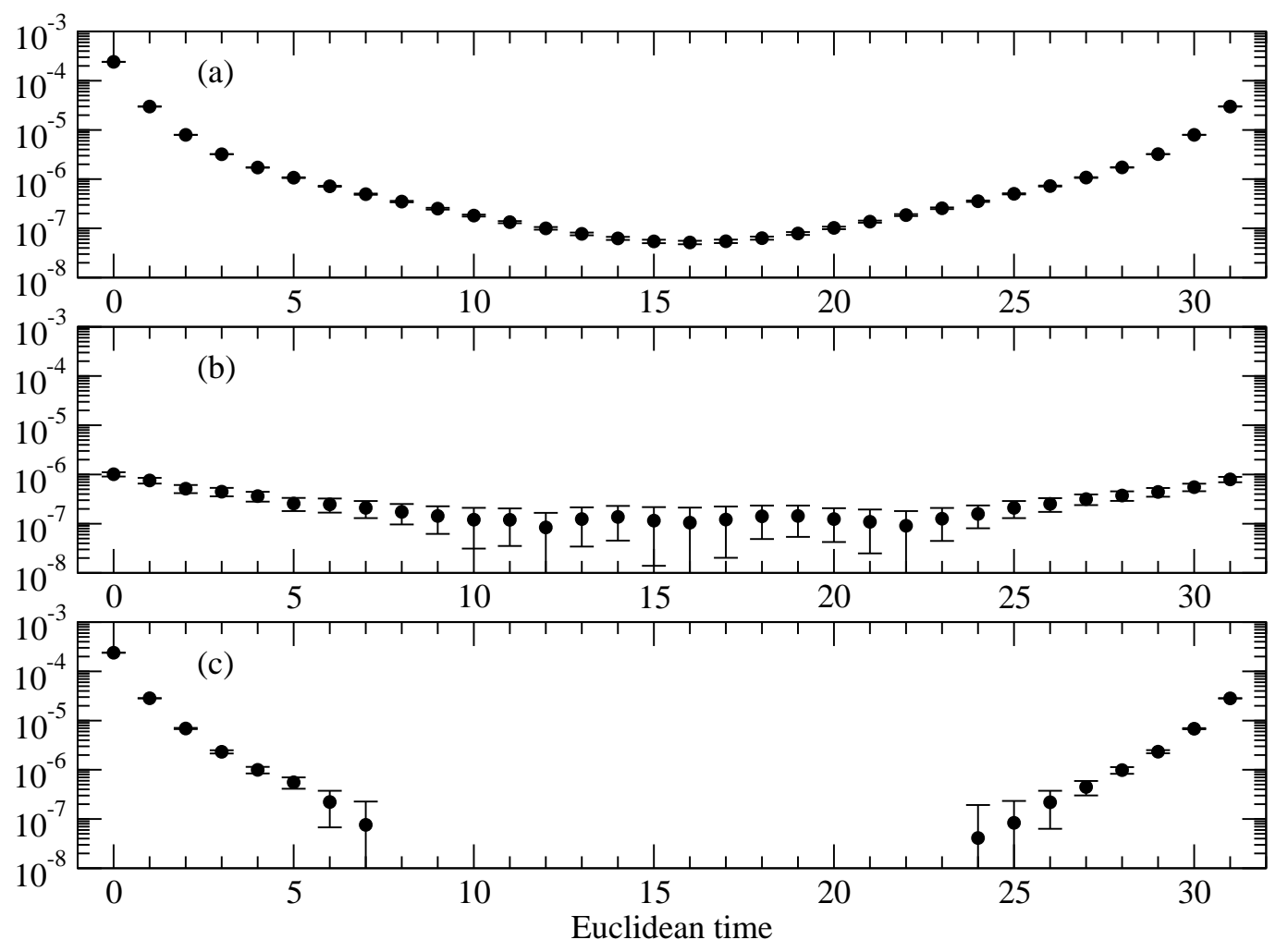

FIG. 8: The (a) connected, (b) disconnected, and (c) total contributions to the correlation function of an isoscalar pseudoscalar meson at $\beta=2.2$ and $m_{0}=-0.75$.

constant, thus justifying the construction of an effective field theory containing only the five Goldstone fields introduced in [24] and then adapted to UMT in [8]. We have established the expected pattern of chiral symmetry breaking in the absence of the electroweak interactions. Typically these interactions tend to destabilize the vacuum (see [38] for a recent study) which can be, however, re-stabilized when extending the technicolor theory to give masses to the SM fermions and to the otherwise massless DM candidate.

One might ask whether a light isoscalar scalar meson is present in this gauge theory, reminiscent of the $\sigma / f_{0}(600)$ meson of QCD, that could be viewed as a composite Higgs boson. The isoscalar scalar channel is a major challenge for lattice simulations [39], and we must leave this question to be the focus of a future study.

In the future it will be very interesting to determine more accurately the mass spectrum of the theory, but there are many additional topics waiting to be explored as well. Here we 
mention three of them. The effects of four-fermion interactions, relevant for a realistic extension of the SM, should be investigated [40]. The extension to nonzero temperature would lead to a characterization of the early universe electroweak phase transition. Knowledge of its order and strength is essential for investigating the possibility of providing correct baryon and asymmetric DM genesis at the electroweak scale [41-43]. The nonzero matter density regime is also useful for asymmetric DM physics [3, 8] given that an asymmetry implies the presence of a nonzero chemical potential. In contrast to QCD, the extension to nonzero matter density is directly possible in lattice simulations of this $S U(2)$ theory. That is because the fermions belong to a pseudoreal representation of the $S U(2)$ gauge group and therefore a chemical potential does not lead to an imaginary action. At nonzero matter density a number of interesting phases can emerge, such as baryon superfluidity (due to the condensation of the diquark technibaryon) and rotational invariance breaking (due to spin-one condensates [44-46] arising from Lorentz symmetry breaking at nonzero chemical potential).

\section{ACKNOWLEDGMENTS}

This work was supported in part by the Natural Sciences and Engineering Research Council (NSERC) of Canada. Computing facilities were provided by the Shared Hierarchical Academic Research Computing Network (SHARCNET: http://www.sharcnet.ca).

\section{Appendix A: Proof of Equation (14)}

The Wilson fermion matrix as obtained from Eq. (21) is

$$
\langle U(y) \bar{U}(x)\rangle^{-1}=\left(4+m_{0}\right) \delta_{x y}-\frac{1}{2} \sum_{\mu}\left(\left(1-\gamma_{\mu}\right) U_{\mu}(y) \delta_{y+\mu, x}+\left(1+\gamma_{\mu}\right) U_{\mu}^{\dagger}(x) \delta_{y, x+\mu}\right) .
$$

The transpose is

$$
\left\langle\left[(U(y) \bar{U}(x)]^{T}\right\rangle^{-1}=\left(4+m_{0}\right) \delta_{x y}-\frac{1}{2} \sum_{\mu}\left(\left(1-\gamma_{\mu}^{T}\right) U_{\mu}^{T}(y) \delta_{y+\mu, x}+\left(1+\gamma_{\mu}^{T}\right) U_{\mu}^{*}(x) \delta_{y, x+\mu}\right) .\right.
$$

Applying the charge conjugation operator $C$ we obtain

$$
C^{-1}\left\langle[U(y) \bar{U}(x)]^{T}\right\rangle^{-1} C=\left(4+m_{0}\right) \delta_{x y}-\frac{1}{2} \sum_{\mu}\left(\left(1+\gamma_{\mu}\right) U_{\mu}^{T}(y) \delta_{y+\mu, x}+\left(1-\gamma_{\mu}\right) U_{\mu}^{*}(x) \delta_{y, x+\mu}\right) .
$$


Recalling that for a general $\operatorname{SU}(2)$ matrix $\mathcal{U}$ the following identity holds:

$$
\left(-i \sigma^{2}\right)^{-1} \mathcal{U}\left(-i \sigma^{2}\right)=\mathcal{U}^{*}
$$

we deduce:

$$
\begin{aligned}
& C^{-1}\left(-i \sigma^{2}\right)^{-1}\left\langle[U(y) \bar{U}(x)]^{T}\right\rangle^{-1} C\left(-i \sigma^{2}\right) \\
= & \left(4+m_{0}\right) \delta_{x y}-\frac{1}{2} \sum_{\mu}\left(\left(1+\gamma_{\mu}\right) U_{\mu}^{+}(y) \delta_{y+\mu, x}+\left(1-\gamma_{\mu}\right) U_{\mu}(x) \delta_{y, x+\mu}\right) .
\end{aligned}
$$

By direct comparison with Eq. (A1) we arrive at

$$
C^{-1}\left(-i \sigma^{2}\right)^{-1}\left\langle[U(y) \bar{U}(x)]^{T}\right\rangle C\left(-i \sigma^{2}\right)=\langle U(x) \bar{U}(y)\rangle
$$

which corresponds to Eq. (14). In the main text we dropped the $\langle.$.$\rangle symbols, indicating$ the vacuum expectation value, to ease the notation.

[1] E. Nardi, F. Sannino and A. Strumia, JCAP 0901, 043 (2009) [arXiv:0811.4153 [hep-ph]].

[2] S. Nussinov, Phys. Lett. B 165, 55 (1985). S. M. Barr, R. S. Chivukula and E. Farhi, Phys. Lett. B 241, 387 (1990).

[3] S. B. Gudnason, C. Kouvaris and F. Sannino, Phys. Rev. D 73, 115003 (2006) arXiv:hepph/0603014]; idem Phys. Rev. D 74, 095008 (2006) [arXiv:hep-ph/0608055].

[4] R. Foadi, M. T. Frandsen and F. Sannino, Phys. Rev. D 80, 037702 (2009) [arXiv:0812.3406 [hep-ph]].

[5] M. Y. Khlopov and C. Kouvaris, Phys. Rev. D 78, 065040 (2008) [arXiv:0806.1191 [astro-ph]].

[6] D. D. Dietrich and F. Sannino, Phys. Rev. D 75, 085018 (2007) [arXiv:hep-ph/0611341].

[7] F. Sannino, Acta Phys. Polon. B 40, 3533 (2009) [arXiv:0911.0931 [hep-ph]].

[8] T. A. Ryttov and F. Sannino, Phys. Rev. D 78, 115010 (2008) [arXiv:0809.0713 [hep-ph]].

[9] D. Hooper, J. March-Russell and S. M. West, Phys. Lett. B 605, 228 (2005) arXiv:hepph/0410114]. C. Kouvaris, Phys. Rev. D 78, 075024 (2008) [arXiv:0807.3124[hep-ph]]. D. E. Kaplan, M. A. Luty and K. M. Zurek, Phys. Rev. D 79, 115016 (2009) arXiv:0901.4117 [hepph]]. L. J. Hall, J. March-Russell and S. M. West, arXiv:1010.0245 [hep-ph]. M. Taoso, J. Phys. Conf. Ser. 259, 012102 (2010). M. R. Buckley and L. Randall, arXiv:1009.0270 [hep-ph]. B. Dutta and J. Kumar, Phys. Lett. B 699, 364 (2011) |arXiv:1012.1341 [hep-ph]]. T. Cohen, 
D. J. Phalen, A. Pierce and K. M. Zurek, Phys. Rev. D 82, 056001 (2010) [arXiv:1005.1655 [hepph]]. A. Falkowski, J. T. Ruderman and T. Volansky, arXiv:1101.4936[hep-ph]. M. T. Frandsen, S. Sarkar and K. Schmidt-Hoberg, arXiv:1103.4350 [hep-ph]. M. L. Graesser, I. M. Shoemaker, L. Vecchi, JHEP 1110, 110 (2011). [arXiv:1103.2771 [hep-ph]]. J. March-Russell, M. McCullough, [arXiv:1106.4319 [hep-ph]]. M. L. Graesser, I. M. Shoemaker, L. Vecchi, [arXiv:1107.2666 [hep-ph]].

[10] M. T. Frandsen and F. Sannino, Phys. Rev. D 81, 097704 (2010) [arXiv:0911.1570 [hep-ph]].

[11] A. Belyaev, M. T. Frandsen, S. Sarkar and F. Sannino, Phys. Rev. D 83, 015007 (2011) [arXiv:1007.4839[hep-ph]].

[12] Z. Ahmed et al. [CDMS-II Collaboration], Phys. Rev. Lett. 106, 131302 (2011) arXiv:1011.2482 [astro-ph.CO]].

[13] J. Angle et al. [XENON10 Collaboration], arXiv:1104.3088 [astro-ph.CO].

[14] E. Aprile et al. [XENON100 Collaboration], arXiv:1104.2549 [astro-ph.CO].

[15] R. Bernabei et al. [DAMA Collaboration], Eur. Phys. J. C 56, 333 (2008) [arXiv:0804.2741 [astro-ph]].

[16] C. E. Aalseth et al. [CoGeNT collaboration], Phys. Rev. Lett. 106, 131301 (2011) [arXiv:1002.4703 [astro-ph.CO]].

[17] M. Y. Khlopov, A. G. Mayorov and E. Y. Soldatov, Int. J. Mod. Phys. D 19, 1385 (2010) [arXiv:1003.1144 [astro-ph.CO]].

[18] D. Tucker-Smith and N. Weiner, Phys. Rev. D 64, 043502 (2001) [arXiv:hep-ph/0101138].

D. Tucker-Smith and N. Weiner, Phys. Rev. D 72, 063509 (2005) [arXiv:hep-ph/0402065].

[19] S. Chang, J. Liu, A. Pierce, N. Weiner and I. Yavin, JCAP 1008, 018 (2010) [arXiv:1004.0697 [hep-ph]].

[20] J. L. Feng, J. Kumar, D. Marfatia and D. Sanford, arXiv:1102.4331 [hep-ph].

[21] M. T. Frandsen, F. Kahlhoefer, J. March-Russell, C. McCabe, M. McCullough and K. SchmidtHoberg, arXiv:1105.3734 [hep-ph].

[22] E. Del Nobile, C. Kouvaris, F. Sannino, [arXiv:1105.5431 [hep-ph]].

[23] G. Angloher, M. Bauer, I. Bavykina, A. Bento, C. Bucci, C. Ciemniak, G. Deuter, F. von Feilitzsch et al., [arXiv:1109.0702 [astro-ph.CO]].

[24] T. Appelquist, P. S. Rodrigues da Silva, F. Sannino, Phys. Rev. D60, 116007 (1999). [hepph/9906555. 
[25] Z. -y. Duan, P. S. Rodrigues da Silva, F. Sannino, Nucl. Phys. B592, 371-390 (2001). [hep$\mathrm{ph} / 0001303]$.

[26] S. Hands, J. B. Kogut, M. -P. Lombardo, S. E. Morrison, Nucl. Phys. B558, 327-346 (1999). [hep-lat/9902034]. J. B. Kogut, M. A. Stephanov, D. Toublan, Phys. Lett. B464, 183-191 (1999). [hep-ph/9906346]. S. Hands, I. Montvay, S. Morrison, M. Oevers, L. Scorzato, J. Skullerud, Eur. Phys. J. C17, 285-302 (2000). [hep-lat/0006018]. R. Aloisio, V. Azcoiti, G. Di Carlo, A. Galante, A. F. Grillo, Phys. Lett. B493, 189-196 (2000). [hep-lat/0009034]. J. B. Kogut, D. K. Sinclair, S. J. Hands, S. E. Morrison, Phys. Rev. D64, 094505 (2001). [hep-lat/0105026]. S. Hands, I. Montvay, M. Oevers, L. Scorzato, J. Skullerud, Nucl. Phys. Proc. Suppl. 94, 461-468 (2001). [hep-lat/0010085]. R. Aloisio, V. Azcoiti, G. Di Carlo, A. Galante, A. F. Grillo, Nucl. Phys. B606, 322-336 (2001). [hep-lat/0011079]. J. B. Kogut, D. Toublan, D. K. Sinclair, Phys. Lett. B514 (2001) 77-87. [hep-lat/0104010]. J. B. Kogut, D. Toublan, D. K. Sinclair, Nucl. Phys. B642, 181-209 (2002). [hep-lat/0205019]. J. B. Kogut, D. Toublan, D. K. Sinclair, Phys. Rev. D68, 054507 (2003). [hep-lat/0305003]. Y. Nishida, K. Fukushima, T. Hatsuda, Phys. Rept. 398, 281-300 (2004). [hep-ph/0306066]. M. -P. Lombardo, M. L. Paciello, S. Petrarca, B. Taglienti, Eur. Phys. J. C58, 69-81 (2008). [arXiv:0804.4863 [hep-lat]].

[27] L. Del Debbio, M. T. Frandsen, H. Panagopoulos, F. Sannino, JHEP 0806, 007 (2008). [arXiv:0802.0891[hep-lat]].

[28] K. Fukushima, JHEP 0807, 083 (2008). [arXiv:0806.1104 [hep-ph]].

[29] H. Matsufuru, Y. Kikukawa, N. Yamada, PoS LAT2009, 064 (2009).

[30] K. -i. Nagai, G. Carrillo-Ruiz, G. Koleva, R. Lewis, Phys. Rev. D80, 074508 (2009). [arXiv:0908.0166 [hep-lat]].

[31] S. Catterall, F. Sannino, Phys. Rev. D76, 034504 (2007). [arXiv:0705.1664 [hep-lat]].

[32] A. J. Hietanen, J. Rantaharju, K. Rummukainen, K. Tuominen, JHEP 0905, 025 (2009). [arXiv:0812.1467[hep-lat]].

[33] S. Hands, P. Sitch, J. -I. Skullerud, Phys. Lett. B662, 405-412 (2008). [arXiv:0710.1966 [hep-lat]].

[34] L. Del Debbio, A. Patella, C. Pica, Phys. Rev. D81, 094503 (2010). |arXiv:0805.2058 [hep-lat]].

[35] J. Foley, K. Jimmy Juge, A. O'Cais, M. Peardon, S. M. Ryan, J. -I. Skullerud, Comput. Phys. Commun. 172, 145-162 (2005). [hep-lat/0505023].

[36] C. Morningstar, J. Bulava, J. Foley, K. J. Juge, D. Lenkner, M. Peardon, C. H. Wong, Phys. Rev. D83, 114505 (2011). arXiv:1104.3870 [hep-lat]]. 
[37] G. M. de Divitiis, R. Frezzotti, M. Guagnelli, M. Masetti, R. Petronzio, Phys. Lett. B367, 279-282 (1996). [hep-lat/9510048]. C. Gattringer, C. B. Lang, Lect. Notes Phys. 788, 1-211 (2010).

[38] T. A. Ryttov, R. Shrock, [arXiv:1107.3572 [hep-ph]].

[39] T. Kunihiro, S. Muroya, A. Nakamura, C. Nonaka, M. Sekiguchi, H. Wada, EPJ Web Conf. 3, 03010 (2010).

[40] H. S. Fukano, F. Sannino, Phys. Rev. D82, 035021 (2010). |arXiv:1005.3340 [hep-ph]].

[41] J. M. Cline, M. Jarvinen, F. Sannino, Phys. Rev. D78, 075027 (2008). [arXiv:0808.1512 [hep-ph]]. See also references therein.

[42] M. Jarvinen, T. A. Ryttov, F. Sannino, Phys. Lett. B680, 251-254 (2009). arXiv:0901.0496 [hep$\mathrm{ph}]$.

[43] M. Jarvinen, T. A. Ryttov, F. Sannino, Phys. Rev. D79, 095008 (2009). [arXiv:0903.3115 [hep$\mathrm{ph}]$.

[44] F. Sannino, Phys. Rev. D67, 054006 (2003). |hep-ph/0211367|.

[45] J. T. Lenaghan, F. Sannino, K. Splittorff, Phys. Rev. D65, 054002 (2002). [hep-ph/0107099].

[46] F. Sannino, W. Schafer, Phys. Lett. B527, 142-148 (2002). [hep-ph/0111098]. 